\title{
Integral points on the elliptic curve $y^{2}=x^{3}+27 x-62$
}

\section{Olcay Karaatlı* and Refik Keskin}

\section{"Correspondence:}

okaraatli@sakarya.edu.tr Department of Mathematics, Sakarya University, Sakarya, 54187, Turkey

\begin{abstract}
We give a new proof that the elliptic curve $y^{2}=x^{3}+27 x-62$ has only the integral points $(x, y)=(2,0)$ and $(x, y)=(28,844,402, \pm 15,491,585,540)$ using elementary number theory methods and some properties of generalized Fibonacci and Lucas sequences.

MSC: 11B25; $11 \mathrm{~B} 37$

Keywords: elliptic curves; integral point; generalized Fibonacci and Lucas sequences
\end{abstract}

\section{Introduction}

Let $P$ and $Q$ be non-zero integers with $P^{2}+4 Q \neq 0$. The generalized Fibonacci sequence $\left(U_{n}(P, Q)\right)$ and the Lucas sequence $\left(V_{n}(P, Q)\right)$ are defined by the following recurrence relations:

$$
U_{0}(P, Q)=0, \quad U_{1}(P, Q)=1, \quad U_{n+2}(P, Q)=P U_{n+1}(P, Q)+Q U_{n}(P, Q) \quad \text { for } n \geq 0
$$

and

$$
V_{0}(P, Q)=2, \quad V_{1}(P, Q)=P, \quad V_{n+2}(P, Q)=P V_{n+1}(P, Q)+Q V_{n}(P, Q) \quad \text { for } n \geq 0
$$

$U_{n}(P, Q)$ is called the $n$th generalized Fibonacci number and $V_{n}(P, Q)$ is called the $n$th generalized Lucas number. Also, generalized Fibonacci and Lucas numbers for negative subscripts are defined as

$$
U_{-n}(P, Q)=\frac{-U_{n}(P, Q)}{(-Q)^{n}} \quad \text { and } \quad V_{-n}=\frac{V_{n}(P, Q)}{(-Q)^{n}} \quad \text { for } n \geq 1 \text {, }
$$

respectively. Taking $\alpha=\left(P+\sqrt{P^{2}+4 Q}\right) / 2$ and $\beta=\left(P-\sqrt{P^{2}+4 Q}\right) / 2$ to be the roots of the characteristic equation $x^{2}-P x-Q=0$, we have the well-known expressions named Binet's formulas

$$
U_{n}(P, Q)=\left(\alpha^{n}-\beta^{n}\right) /(\alpha-\beta) \quad \text { and } \quad V_{n}(P, Q)=\alpha^{n}+\beta^{n}
$$

for all $n \in \mathbb{Z}$. Instead of $U_{n}(P, Q)$ and $V_{n}(P, Q)$, we use $U_{n}$ and $V_{n}$, respectively. For $P=$ $Q=1$, the sequence $\left(U_{n}\right)$ is the familiar Fibonacci sequence $\left(F_{n}\right)$ and the sequence $\left(V_{n}\right)$ is the familiar Lucas sequence $\left(L_{n}\right)$. If $P=2$ and $Q=1$, then we have the well-known Pell sequence $\left(P_{n}\right)$ and Pell-Lucas sequence $\left(Q_{n}\right)$. For $Q=-1$, we represent $\left(U_{n}\right)$ and $\left(V_{n}\right)$ by

\section{Springer}

(c) 2013 Karaatlı and Keskin; licensee Springer. This is an Open Access article distributed under the terms of the Creative Commons Attribution License (http://creativecommons.org/licenses/by/2.0), which permits unrestricted use, distribution, and reproduction in any medium, provided the original work is properly cited. 
$\left(u_{n}\right)$ and $\left(v_{n}\right)$, respectively. Thus $u_{0}=0, u_{1}=P$ and $u_{n+1}=P u_{n}-u_{n-1}$ and $v_{0}=2, v_{1}=P$ and $v_{n+1}=P v_{n}-v_{n-1}$ for all $n \geq 1$. Also, it is seen from Eq. (1.1) that

$$
u_{-n}=-u_{n}(P,-1) \quad \text { and } \quad v_{-n}=v_{n}(P,-1)
$$

for all $n \geq 1$. For more information about generalized Fibonacci and Lucas sequences, one can consult [1-5].

There has been much interest in determining the problem of the integral points on elliptic curves, and many advanced methods have been developed to solve such problems (see [6, 7] and [8]). In 1987, Don Zagier [9] proposed that the largest integral point on the elliptic curve

$$
y^{2}=x^{3}+27 x-62
$$

is $(x, y)=(28,844,402, \pm 154,914,585,540)$. Then the same problem was dealt with by some authors. In [10], Zhu and Chen found all integral points on (1.3) by using algebraic number theory and $p$-adic analysis. In [11], Wu proved that (1.3) has only the integral points $(x, y)=(2,0)$ and $(28,844,402, \pm 154,914,585,540)$ using some results of quartic Diophantine equations with elementary number methods. After that, in [12], the authors found the integral points on (1.3) using similar methods to those given in [11]. In this paper, we determine that the largest integral point on the elliptic curve $y^{2}=x^{3}+27 x-62$ is $(x, y)=(28,844,402, \pm 154,914,585,540)$ by using elementary number theory methods and some properties of generalized Fibonacci and Lucas sequences. Our proof is extremely different from the proofs of the others.

\section{Preliminaries}

In this section, we present two theorems and some well-known identities regarding the sequences $\left(u_{n}\right)$ and $\left(v_{n}\right)$, which will be useful during the proof of the main theorem.

We state the following theorem from [13].

Theorem 2.1 Let $P>2$. If $u_{n}=c x^{2}$ with $c \in\{1,2,3,6\}$ and $n>3$, then $(n, P, c)=(4,338,1)$ or $(6,3,1)$.

The following theorem is a well-known theorem (see [14]).

Theorem 2.2 Let $m \geq 1$ and $n \geq 1$. Then $\left(u_{m}, u_{n}\right)=u_{(m, n)}$.

The well-known identities for $\left(u_{n}\right)$ and $\left(v_{n}\right)$ are as follows:

$$
\begin{aligned}
& u_{2 n}=u_{n} v_{n}, \\
& v_{n}=u_{n+1}-u_{n-1}, \\
& u_{2 k+1}-1=u_{k} v_{k+1} .
\end{aligned}
$$

Moreover, if $P$ is even, then

$$
\begin{aligned}
& u_{n} \text { is even } \Leftrightarrow n \text { is even, } \\
& u_{n} \text { is odd } \Leftrightarrow n \text { is odd. }
\end{aligned}
$$




\section{Proof of the main theorem}

The main theorem we deal with here is as follows.

Theorem 3.1 The elliptic curve $y^{2}=x^{3}+27 x-62$ has only the integral points $(x, y)=(2,0)$ and $(28,844,402, \pm 154,914,585,540)$.

Proof Assume that $(x, y)$ is an integral point on the elliptic curve $y^{2}=x^{3}+27 x-62$. It can be easily seen that $x>0$. On the other hand, obviously, the elliptic curve $y^{2}=x^{3}+27 x-62$ has only the integral point $(x, y)=(2,0)$ with $y=0$. Hence, we may assume that $y \neq 0$. Let $k=x-2$. Substituting this value of $k$ into $y^{2}=x^{3}+27 x-62$, we get

$$
y^{2}=k\left(k^{2}+6 k+39\right) \text {. }
$$

Since $y \neq 0$, it is obvious that $y^{2}>0$. On the other hand, since $k^{2}+6 k+39=(k+3)^{2}+30>0$, we conclude that $k>0$. Clearly, $d=\left(k, k^{2}+6 k+39\right)=1,3,13$ or 39 . So, we get from (3.1) that

$$
k=d a^{2}, \quad k^{2}+6 k+39=d b^{2}, \quad y= \pm d a b
$$

for some positive integers $a$ and $b$.

If $d=1$, then from (3.2) we get $a^{4}+6 a^{2}+39=b^{2}$. Completing the square gives $\left(a^{2}+3\right)^{2}+$ $30=b^{2}$. This implies that $\left[b-\left(a^{2}+3\right)\right]\left[b+\left(a^{2}+3\right)\right]=30$. It can be easily shown that there are no integers $a$ and $b$ satisfying the previous equation.

If $d=3$, then from (3.2) we obtain $9 a^{4}+18 a^{2}+39=3 b^{2}$. Completing the square gives

$$
b^{2}-3\left(a^{2}+1\right)^{2}=10 \text {. }
$$

Working on modulo 8 shows that (3.3) is impossible.

If $d=13$, then from (3.2) we immediately have $169 a^{4}+78 a^{2}+39=13 b^{2}$. Completing the square gives

$$
\left(13 a^{2}+3\right)^{2}-13 b^{2}=-30
$$

Working on modulo 8 shows that (3.4) is impossible.

Lastly, we consider (1.3) for the case when $d=39$. If $d=39$, then from (3.2) we get $k=$ $39 a^{2}$ and $k^{2}+6 k+39=39 b^{2}$. Substituting $k=39 a^{2}$ into $k^{2}+6 k+39=39 b^{2}$ and completing the square give

$$
\left(39 a^{2}+3\right)^{2}+30=39 b^{2} .
$$

This equation is of the form

$$
u^{2}-39 v^{2}=-30 \text {. }
$$

Let $x_{n}+y_{n} \sqrt{39}$ be a solution of the equation $x^{2}-39 y^{2}=1$. Since the fundamental solution of this equation is $\alpha=25+4 \sqrt{39}$, we get $x_{n}+y_{n} \sqrt{39}=\alpha^{n}$, and therefore $x_{n}=\left(\alpha^{n}+\beta^{n}\right) / 2$ 
and $y_{n}=\left(\alpha^{n}-\beta^{n}\right) / 2 \sqrt{39}$, where $\beta=25-4 \sqrt{39}$. It can be easily seen that $x_{n}=v_{n}(50,-1) / 2$ and $y_{n}=4 u_{n}(50,-1)$. Equation (3.6) has exactly two solution classes and the fundamental solutions are $3+\sqrt{39}$ and $3-\sqrt{39}$. So, the general solution of (3.6) is given by

$$
\begin{aligned}
& a_{n}+b_{n} \sqrt{39}=(3-\sqrt{39})\left(x_{n}+y_{n} \sqrt{39}\right), \\
& a_{n}+b_{n} \sqrt{39}=(3+\sqrt{39})\left(x_{n}+y_{n} \sqrt{39}\right),
\end{aligned}
$$

with $n \geq 1$, respectively [15]. Considering first Eq. (3.7), we readily obtain $a_{n}=3 x_{n}-39 y_{n}$. Since $x_{n}=v_{n} / 2$ and $y_{n}=4 u_{n}$, it follows that

$$
a_{n}=\left(3 v_{n}-312 u_{n}\right) / 2 .
$$

From (2.2), if we write $u_{n+1}-u_{n-1}$ instead of $v_{n}$ and rearrange the above equation, then we get $a_{n}=-81 u_{n}-3 u_{n-1}$. This means that $39 a^{2}+3=-81 u_{n}-3 u_{n-1}$ by (3.5). Dividing both sides of the equation by 3 gives $13 a^{2}+1=-27 u_{n}-u_{n-1}$. However, this is impossible for $13 a^{2}+1>0$ and $n \geq 1$. Another possibility is that $-39 a^{2}-3=-81 u_{n}-3 u_{n-1}$, implying that

$$
13 a^{2}+1=27 u_{n}+u_{n-1}
$$

It can be shown by the induction method that

$$
u_{n} \equiv \begin{cases}-n(\bmod 13) & \text { if } n \text { is even } \\ n(\bmod 13) & \text { if } n \text { is odd }\end{cases}
$$

and

$$
u_{n} \equiv n(\bmod 8)
$$

So, working on modulo 8 and using (3.11) in Eq. (3.9) lead to a contradiction.

Now, we consider Eq. (3.8). Then we immediately have $a_{n}=3 x_{n}+39 y_{n}$. Since $x_{n}=v_{n} / 2$ and $y_{n}=4 u_{n}$, it follows that $a_{n}=\left(3 v_{n}+312 u_{n}\right) / 2$. In view of (2.2), we readily obtain $a_{n}=$ $3 u_{n+1}+81 u_{n}$. By (3.5), we get $39 a^{2}+3=3 u_{n+1}+81 u_{n}$, implying that

$$
13 a^{2}+1=u_{n+1}+27 u_{n}
$$

Assume that $n$ is odd. By using (3.10), we get

$$
u_{n+1}+27 u_{n} \equiv-n-1+27 n \equiv-1(\bmod 13)
$$

a contradiction by (3.12). So, $n$ is even. Now, let us assume that $a$ is odd in Eq. (3.12). Then using (3.11) gives

$$
u_{n+1}+27 u_{n} \equiv n+1+3 n \equiv 4 n+1 \equiv 6(\bmod 8) \text {, }
$$

i.e.,

$$
4 n \equiv 5(\bmod 8)
$$


which is impossible. So, $a$ is even, and therefore $a=2 m$ for some positive integer $m$. Substituting $a=2 m$ into (3.12), we get

$$
52 m^{2}+1=u_{n+1}+27 u_{n}
$$

In the above equation, if $m$ is odd, then from (3.11) we get

$$
u_{n+1}+27 u_{n} \equiv n+1+3 n \equiv 4 n+1 \equiv 5(\bmod 8)
$$

which implies that

$$
n \equiv 1(\bmod 2)
$$

But this is impossible since $n$ is even. As a consequence, $m$ is even and therefore we conclude that $4 \mid a$. We now return to (3.12). Since $n$ is even, $n=2 r$ for some $r>0$. Then (3.12) becomes

$$
13 a^{2}=u_{2 r+1}-1+27 u_{2 r}
$$

By (2.3) and (2.1), it can be seen that $u_{2 r+1}-1+27 u_{2 r}=u_{r} v_{r+1}+27 u_{r} v_{r}=u_{r}\left(v_{r+1}+27 v_{r}\right)$ and therefore

$$
13 a^{2}=u_{r}\left(v_{r+1}+27 v_{r}\right) .
$$

By using (2.2), we get $13 a^{2}=u_{r}\left(u_{r+2}-u_{r}+27 u_{r+1}-27 u_{r-1}\right)$. In view of the recurrence relation of the sequence $u_{r}$, we immediately have

$$
13 a^{2}=u_{r}\left(3,848 u_{r}-104 u_{r-1}\right) .
$$

Dividing both sides of the above equation by 13 and rearranging the equation gives

$$
a^{2}=8 u_{r}\left(37 u_{r}-u_{r-1}\right)
$$

Since $4 \mid a$, it follows that

$$
2(a / 4)^{2}=u_{r}\left(37 u_{r}-u_{r-1}\right)
$$

By Theorem 2.2, since $\left(u_{r}, u_{r-1}\right)=1$, clearly, $\left(u_{r}, 37 u_{r}-u_{r-1}\right)=1$. This implies that either

$$
37 u_{r}-u_{r-1}=2 c^{2}
$$

or

$$
u_{r}=2 c^{2}
$$

for some positive integer $c$, where $u_{r}=u_{r}(50,-1)$. By (2.4) and (2.5), it can be seen that $37 u_{r}-u_{r-1}$ is always odd. Therefore (3.14) is impossible. By Theorem 2.1, (3.15) is impossible for the case when $r>3$. Hence, we have $r \leq 3$. On the other hand, since $u_{r}=2 c^{2}$ is 
even, from (2.4), it follows that $r$ is even. Since $r$ is even and $n=2 r$, we get $n=4$. Substituting this value of $n$ into (3.12), we obtain

$$
13 a^{2}+1=u_{5}+27 u_{4}
$$

Since $u_{5}=6,242,501$ and $u_{4}=124,900$, a simple computation shows that $a=860$. Moreover, since $k=39 a^{2}$ and $x=k+2$, we get $k=28,844,400$ and therefore $x=28,844,402$. Substituting $x=28,844,402$ into $y^{2}=x^{3}+27 x-62$ gives $y= \pm 15,491,585,540$. Hence, the theorem is proved, the elliptic curve $y^{2}=x^{3}+27 x-62$ has only the integral points $(x, y)=(2,0)$ and $(x, y)=(28,844,402, \pm 15,491,585,540)$, which is the largest integral point on it. This completes the proof of the main theorem.

\section{Competing interests}

The authors declare that they have no competing interests.

\section{Authors' contributions}

Both authors contributed equally in the preparation of this article. Both authors read and approved the final manuscript.

\section{Acknowledgements}

Dedicated to Professor Hari M Srivastava.

Received: 9 January 2013 Accepted: 6 March 2013 Published: 2 May 2013

\section{References}

1. Kalman, D, Mena, R: The Fibonacci numbers-exposed. Math. Mag. 76, 167-181 (2003)

2. Karaatlı, O, Keskin, R: On some diophantine equations related to square triangular and balancing numbers. J. Algebra, Number Theory: Adv. Appl. 4(2), 71-89 (2010)

3. Muskat, JB: Generalized Fibonacci and Lucas sequences and rootfinding methods. Math. Comput. 61, 365-372 (1993)

4. Rabinowitz, S: Algorithmic manipulation of Fibonacci identities. In: Application of Fibonacci Numbers, vol. 6, pp. 389-408. Kluwer Academic, Dordrecht (1996)

5. Ribenboim, P: My Numbers, My Friends. Springer, New York (2000)

6. Baker, A: The Diophantine equation $y^{2}=a x^{3}+b x^{2}+c x+d$. J. Lond. Math. Soc. 43, 1-9 (1968)

7. Stroeker, RJ, Tzanakis, N: On the elliptic logarithm method for elliptic Diophantine equations: reflections and an improvement. Exp. Math. 8, 135-149 (1999)

8. Stroeker, RJ, Tzanakis, N: Computing all integer solutions of a genus 1 equation. Math. Comput. 72, 1917-1933 (2003)

9. Zagier, D: Large integral points on elliptic curves. Math. Comput. 48, 425-436 (1987)

10. Zhu, H, Chen, J: Integral points on $y^{2}=x^{3}+27 x-62$. J. Math. Study 42(2), 117-125 (2009)

11. $\mathrm{Wu}, \mathrm{H}$ : Points on the elliptic curve $y^{2}=x^{3}+27 x-62$. Acta Math. Sin., Chin. Ser. 53(1), 205-208 (2010)

12. He, Y, Zhang, W: An elliptic curve having large integral points. Czechoslov. Math. J. 60(135), 1101-1107 (2010)

13. Mignotte, M, Pethő, A: Sur les carrés dans certanies suites de Lucas. J. Théor. Nr. Bordx. 5(2), 333-341 (1993)

14. Ribenboim, P: An algorithm to determine the points with integral coordinates in certain elliptic curves. J. Number Theory 74, 19-38 (1999)

15. Nagell, T: Introduction to Number Theory. Wiley, New York (1981) 